\title{
An Experimental Model to Induce Homogeneous and Progressive Pulmonary Fibrosis in Rats
}

\author{
Syed Mohammad Abdullah, Papiya Mitra Mazumder* \\ Department of Pharmaceutical Sciences and Technology, Birla Institute of Technology-Mesra, Ranchi, Jharkhand, INDIA.
}

\begin{abstract}
Background: This study aims to develop a new experimental model of pulmonary fibrosis (PF) as an alternative to conventional bleomycin model to overcome its limitations and effectively screened the therapeutic agents. Similarity of new experimental model to human lung fibrosis particularly as uniform and continuous progression has encouraged us to evaluate the usefulness of this system by assessment of anti-fibrotic drug pirfenidone parallel with bleomycin induced PF. Materials and Methods: In new model, ovalbumin (OVA) related asthma induced rats were set to inhaled nebulized formaldehyde solution $(0.5 \% \mathrm{v} / \mathrm{v}, 30 \mathrm{~min})$ subsequent with cigarettes smoke exposure twice daily for 14,28 or 42 days and designated as AFC treated rats. However, bleomycin (BLM) treated rats were administered $(5 \mathrm{lU} / \mathrm{kg}$ ) single dose intratracheal injection. The pulmonary associated effects were assessed through cells permeation study, biochemical evaluation, physical and physiological parameters, survival analysis and histopathological examination. Results: Overall, pulmonary associated effects induced by new as well as conventional bleomycin model exhibited almost similar results. Though, new model developed homogeneous and time dependent advancement in pulmonary severity with enhanced survival ratio as compared to bleomycin model. Comparatively, new model also represented low extent of normalization with pirfenidone treatment due to establishment of progressive fibrosis. Conclusion: This study reflects the role of formaldehyde and cigarette smoke in reinforcement of PF particularly during the lung inflammatory condition. Also, it provides novel idea to induce homogeneous and progressive PF in rats, which could resembles human lung fibrosis more specifically than conventionally induced by intratracheal bleomycin instillation.
\end{abstract}

Key words: Asthma, Cigarette smoke, Experimental model, Formaldehyde, Pulmonary fibrosis.

\section{INTRODUCTION}

Organ fibrosis is a pathological condition manifested by surplus production of protein (extracellular matrix) and subsequent deposition within interstitial tissues. By considering different organs, lungs would be considered as the most vulnerable to develop fibrosis. ${ }^{1}$ Generally, polluted air, occupational airborne particles and chemical fumes insults may provoke interstitial lung disease. ${ }^{2}$ Among various forms of interstitial lung disease, pulmonary fibrosis (PF) is considered as the most frequent type. ${ }^{3}$ Where, PF causes persistent lung deterioration leading to death because of deprived prognosis and continuous decline in respiratory functions. ${ }^{4}$ Till now, a number of risk factors related to PF have been discussed in the scientific literatures. Cumulative evidence of several observational studies suggest that air pollution, cigarette smoking and lung inflammatory disease (asthma/ chronic obstructive pulmonary disease) could be considered as significant risk factors in PF. 2,3,5,6 However, due to its unknown etiology and pathogenesis neither ideal in vivo model has been established nor effective therapeutic agents have been developed. ${ }^{7}$
Submission Date: 21-08-2020; Revision Date: 14-12-2020; Accepted Date: 09-08-2021

DOI: 10.5530/ijper.55.3s.182 Correspondence:

Dr. Papiya Mitra Mazumder, Department of Pharmaceutical Sciences and Technology, Birla Institute of Technology-Mesra, Ranchi, Jharkhand-835215, INDIA. Phone: +91-9431327044,

Email - pmitramazumder@ bitmesra.ac.in

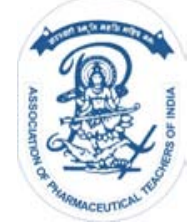

www.ijper.org 
Including different variety of experimental models, $\mathrm{PF}$ induced by single dose bleomycin intratracheal instillation in rodents has been extensively used to evaluate the efficacy of various anti-fibrotic agents. However, this conventional model has several limitations ${ }^{8}$ such as, a neck surgery is required which, may affect animal sustainability and food intake activity. In consequences, it is difficult to justify the reason behind the causality and body weight reduction particularly during $1^{\text {st }}$ week after drug instillation. Moreover, bleomycin model is unable to reveal the exact mechanisms involved behind the onset of PF. ${ }^{9}$ Further, it is expensive as various supporting and imaging instruments are needed during intratracheal instillation.

Nowadays, it has been observed that PF incidence continuously rises around the world particularly where, air-pollution is a major concern. ${ }^{10}$ The foremost components contributing as air pollutant are usually hydrocarbons. ${ }^{11}$ However, photochemical oxidation and incomplete combustion of hydrocarbons generates formaldehyde, which is ubiquitously present in atmosphere. ${ }^{12}$ Its frequently inhalation causes irritation to the epithelial cells of respiratory system. Also, it acts as potent elicitor to induced inflammation in lower airways and lungs. ${ }^{13}$ Moreover, formaldehyde inhalation promotes neutrophils infiltration and exaggerates collagen synthesis in lungs of bleomycin-treated rats. ${ }^{14}$ Furthermore, inhalation of formaldehyde enabled to produce pneumonitis by enhancing the oxidative-stress in the lungs of rats. ${ }^{15}$ Another in vivo study reveals, cigarette smoking intensifies oxidative-stress and deteriorating condition of lungs in bleomycin administrated mice. ${ }^{16}$ Subsequently, a clinical study represented inhalation of formaldehyde triggers sensitization and amplifies the inflammatory condition of lungs in asthmatic patients. ${ }^{17}$

Several researchers proposed that oxidative-stress and inflammation could act as significant driving force in creating the imbalance between tissue injuries and repairing process, which might be considered as the basis for the initiation of organ fibrosis. ${ }^{18}$ Accordingly, the fibrosis stimulation by creating disturbance in lung's tissues homoeostasis through enhancing oxidative-stress on one hand and inducing inflammatory conditions on other hand could possibly initiate PF. Therefore, to test this hypothesis, the combined effect of repeated pulmonary insult through formaldehyde inhalation and cigarette smoke exposure in ovalbuminderived asthmatic condition was examined in rats. Also, in view to develop a new experimental PF model, pulmonary effect of combined risk factors and bleomycin insult were assessed for different time duration. In addition, efficacy of pirfenidone was also investigated against these experimental models.

\section{MATERIALS AND METHODS}

\section{Chemicals}

Bleomycin sulphate was purchased as Bleocip-injection manufactured by Cipla Ltd. (Maharashtra, India); Pirfenidone was procured as gift sample from Chemca Drugs Pvt. Ltd. (Andhra Pradesh, India); Ovalbumin, formaldehyde, chloramine-T, hydroxyproline, o-dianisidine dihydrochloride, staining agents were obtained from HiMedia Laboratories Pvt. Ltd. (Mumbai, India); Commercial cigarettes of particular brand was purchased from the local market (Jharkhand, India). All other chemicals were used of high purity and commercially available.

\section{Animals}

Healthy, male albino Wistar rats (Swiss-strain) of aged 10 to 12 weeks (weight: 275 to $325 \mathrm{~g}$ ), were procured from the animal house facility, Birla Institute of Technology-Mesra, Ranchi, Jharkhand, India. They were kept under standard laboratory conditions and acclimatized for 7 days prior to involvement in the study. The study protocol was approved by the committee for the purpose of control and supervision of experiments on animals (CPCSEA) having registration no. 1968/PO/Re/S/17CPCSEA.

\section{Treatments}

All study animals were randomly divided into two groups, with survival surgery (SS) at neck region on dorsal side and with no survival surgery (NSS). In SS group, all animals were subjected to administered single dose intratracheal bleomycin injection (5 IU/ $/ \mathrm{kg}$ ) and were designated as BLM treated rats. However, within NSS-group all animals were subjected to receive successive pulmonary insult through formaldehyde and cigarette smoke post asthma induction and were designated as AFC treated rats. However, in both groups saline treated rats were considered as control and were designated as CSS and CNSS, respectively. Further, within SS and NSS group rats were divided into different arms (5 animals each) as per the duration of study and were treated as follows:

\section{SS (Group-I)}

Arm-I (CSS): $0.5 \mathrm{ml} \mathrm{NS}$ (0.9\% w/v), (i.t) inj., single dose on day 0 and sacrificed after 21 days.

Arm-II (BLM-7); Arm-III (BLM-14); Arm-IV (BLM-21) and Arm-V (BLM-28): $5 \mathrm{IU} / \mathrm{kg}$ bleomycin dissolved in 
$0.5 \mathrm{ml} \mathrm{NS}(0.9 \% \mathrm{w} / \mathrm{v})$, (i.t) inj., single dose on day 0 and sacrificed after 7, 14, 21 and 28 days respectively.

Arm-VI (BLM+PD-21): $5 \mathrm{IU} / \mathrm{kg}$ bleomycin dissolved in $0.5 \mathrm{ml} \mathrm{NS}(0.9 \% \mathrm{w} / \mathrm{v})$, (i.t) inj., single dose on day 0 and then treated with $50 \mathrm{mg} / \mathrm{kg}$ pirfenidone dissolved in $1.0 \mathrm{ml} \mathrm{NS}(0.9 \% \mathrm{w} / \mathrm{v})$, per oral (p.o), once daily (o.d) and sacrificed after 21 days.

\section{NSS (Group-II)}

Arm-I (CNSS): Inhaled NS (0.9\% w/v), $30 \mathrm{~min}$, twice daily (b.d) for 42 days and then sacrificed.

Arm-II (AFC-14); Arm-III (AFC-28); Arm-IV (AFC-42): Asthmatic animals were inhaled formaldehyde solution (FS $0.5 \% \mathrm{v} / \mathrm{v}$ in NS $0.9 \% \mathrm{w} / \mathrm{v}$ ) for $30 \mathrm{~min}$ followed by five (05) cigarettes smoke (CS) exposure, twice daily (b.d) for 14, 28 and 42 days and then sacrificed respectively. Arm-V (AFC+PD-42): Asthmatic animals were inhaled formaldehyde solution (FS $0.5 \% \mathrm{v} / \mathrm{v}$ in NS $0.9 \% \mathrm{w} / \mathrm{v}$ ) for $30 \mathrm{~min}$ followed by five (05) cigarettes smoke (CS) exposure, twice daily (b.d) for 42 days and parallel treated with $50 \mathrm{mg} / \mathrm{kg}$ pirfenidone (PD) dissolved in $1.0 \mathrm{ml} \mathrm{NS}(0.9 \% \mathrm{w} / \mathrm{v})$, per oral (p.o), once daily (o.d) and sacrificed after 42 days.

The doses of $\mathrm{BLM},{ }^{19} \mathrm{PD}^{20}$ and OVA-Al $(\mathrm{OH})_{3}{ }^{21}$ used in the present study were selected on the basis of previous studies. However, dose of formaldehyde solution (FS) and cigarettes smoke (CS) were derived from our dose optimization study.

\section{Study Design}

In SS-group, all animals went for survival-surgery under anesthesia (chloral hydrate $300 \mathrm{mg} / \mathrm{kg}$, i.p) at neck region from dorsal side and small incision was made to expose out the trachea. All animals were led on $60^{\circ}$ inclined supports in upright position and injected (i.t) single dose $\left.0.5 \mathrm{ml} \mathrm{BLM} \mathrm{(5} \mathrm{IU} \mathrm{kg}^{1}\right)$ in saline. In CSS arm, animals were received $0.5 \mathrm{ml}$ saline solution (i.t). After drug administration, skin was sutured and povidone-iodine ointment was applied for quick healing of surgery.

In NSS-group, all animals were administered $0.5 \mathrm{ml}$ OVA-Al $(\mathrm{OH})_{3}$ emulsion (i.p) on three subsequent weeks followed by three successive challenges with nebulized $1 \% \mathrm{w} / \mathrm{v}$ OVA in saline on daily basis (Figure 1). However, control animals designated to CNSS arm were treated with saline solution in similar way. To ensure the onset of asthmatic condition in rats prior to set for FS inhalation and CS exposure, they were examined as per the method discussed in earlier studies. ${ }^{22,23}$ Briefly, rats were examined for decrease in rectal temperature by using digital thermometer (HICKS Thermometers Ltd, India) within first $2 \mathrm{~h}$ and decrease in locomotors

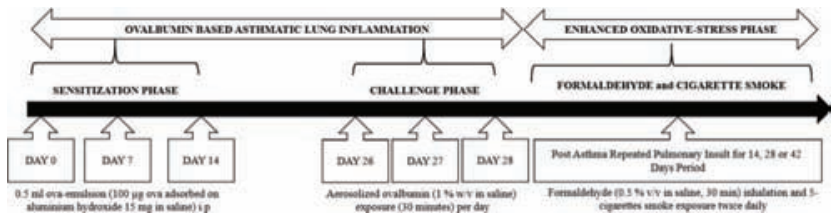

Figure 1: A study design for successive pulmonary insult through combined risk factors (AFC) in experimental rats. (AFC $=$ asthma + formaldehyde + cigarette smokes).

activity (distance travelled and rearing phenomenon recorded for 15 min by using video tracking devices within confined area) within next $2 \mathrm{~h}$, after last OVA-challenge and compared with baseline values. In addition, blood samples were taken out through retro-orbital route to measure the eosinophil count in each rat prior and post challenges, as a marker of allergic condition. After ensuring the induction of asthmatic condition, rats were introduced inside the pixel-glass inhalation chamber of fixed volume $(12 \mathrm{~L})$ and dimension $(1 \times b \times h=27 \times 22 \times 20.2 \mathrm{~cm})$. The chamber was installed with automated compressor nebulizer (OMRON, Model: NE-C28-E, Healthcare Co., Ltd. Kyoto Japan) and air suction pump (HSH-Flo, Model: KYK50BPM, Keyukang Electronic Co., Ltd. China) connected with cigarette ignition holder and both had nominal flow rate: $8 \mathrm{~L} / \mathrm{min}$. In which, within the chamber experimental rats were forced to inhale $0.5 \% \mathrm{v} / \mathrm{v}$ aerosolized $\mathrm{FS}$ for $30 \mathrm{~min}$ and subsequently taken exposure of 05 CS twice daily (morning and evening) for 14, 28, or 42 days. During the experiment, all the study animals (05) of each arm were kept together inside the pixel-glass inhalation chamber.

At the end of study, all rats were sacrificed by decapitation method under mild anesthesia using chloral hydrate $300 \mathrm{mg} / \mathrm{kg}$, i.p and immediately chest was open surgically and bronchoalveolar lavage-fluid (BALF) was collected. The whole lung was harvested from the body and divided into two halves, left and right portions. The right portion was utilized to prepare the lung-homogenate as per the protocol discussed elsewhere and left portion was kept in $10 \%(\mathrm{v} / \mathrm{v})$ formaldehyde solution for histopathological examination. Throughout the study, for biochemical assessment, a spectrophotometer was used to determine the absorbance and the protein concentration in all samples were estimated according to the method of Bradford. ${ }^{24}$

\section{Percent change in body weight}

Body weight of each animal was weighed initially on day 0 and at every week intervals till end of the study. On day 0 , the mean body weight of all study animals was considered as $100(\%)$. The percent change in the 
body weight in each arm was calculated on weekly basis with respect to initial value obtained on day 0 .

\section{Lung/body weight (L/B) ratio}

The complete harvested lung and whole body were weighed in $\mathrm{mg}$ and $\mathrm{g}$ respectively $\mathrm{y}^{25}$ for the calculation of the $\mathrm{L} / \mathrm{B}$ ratio and the $\mathrm{L} / \mathrm{B}$ ratio was expressed as $\mathrm{mg} / \mathrm{g}$ unit.

\section{Exercise performance test}

To perform the physical exercise test in rats, an indigenously assembled forced running wheel apparatus of total inner circumference $1 \mathrm{~m}$ was used and adjusted with electric motor to provide controlled rotational speed to the wheel. Exercise Performance Test (EPT) was conducted by placing a single animal inside the forced running wheel apparatus to assess individual's physical exercise ability which is indirect analogous to lung condition. ${ }^{26}$ Initially, all study animals were compulsorily indulged in 5 min training session at not more than $10 \mathrm{rpm}$ on daily basis for a week to learned exercise and to minimized presentation errors during actual test procedure. For the EPT, the animal was kept within apparatus and motorized wheel was set at constant rotational speed $8 \mathrm{rpm}$ in particular direction. Fixed rotating wheel compelled the animals to maintain their running pace accordingly on regular moving surface until they completely exhausted and unable to remained in erect position inside the apparatus. EPT procedure was conducted for each rat in all arms on the last day of the study.

EPT was evaluated on the basis of distance covered derived by multiplying the constant rotational speed $(\mathrm{rpm})$ with running duration $(\mathrm{min})$ and inner circumference $(\mathrm{m})$ of wheel. Where, the EPT expressed and calculated as follows:

Distance covered $(\mathrm{m})=\frac{\text { Rotation }}{\mathrm{min}} \times$ Running duration $(\mathrm{min}) \times$ Wheel circumference $(\mathrm{m})$

\section{Cells permeation study}

BALF collection from each sacrificed animal was performed, pooled and concentrated by centrifugation at $1000 \mathrm{~g}$. Supernatant of BALF was decanted and stored at $-80^{\circ} \mathrm{C}$ for biochemical estimation and the cells pellet was re-suspended in PBS for total cells count using haemocytometer. However, for differential cells count, BALF sample was gently spread over the glass slide to prepare a thin-smear followed by Giemsa's staining and a total of 300 cells were counted. . $^{27,28}$

\section{Biochemical assessment of BALF}

The total protein (TP) content in BALF (supernatant) was estimated by using Bradford reagent in PBS ( $\mathrm{pH}$ 7.4) and the values are expressed as $\mu \mathrm{g} \cdot \mathrm{ml}^{-1}$. Also, in BALF (supernatant) the creatine kinase-MB (CK-MB), alkaline phosphatase (ALP) and lactate dehydrogenase (LDH) activities $^{19,29}$ were determined by using bioassay kits procured from Coral Clinical Systems (Goa, India) and the values are expressed as U.L-1. Additionally, tumor necrosis factor-alpha (TNF- $\alpha$ ) level ${ }^{28}$ was detected in BALF (supernatant) through rat specific ELISA kit obtained from eBioscience, Inc. (San Diego., USA) and the values are expressed as pg. $\mathrm{ml}^{-1}$.

\section{Biochemical assessment of lung tissue}

The lung-homogenate for the biochemical evaluation was prepared in $1: 10(\mathrm{w} / \mathrm{v})$ ratio in freshly available PBS ( $\mathrm{pH}$ 7.4) by using Teflon-glass homogenizer and centrifuged the whole content at 10,000 rpm at least for $20 \mathrm{~min}$ at $4^{\circ} \mathrm{C}$. Then, clear supernatant was taken out for the assessment of oxidative-stress markers. Superoxide dismutase (SOD) activity was evaluated by the inhibition in the reduction rate of nitro blue tetrazolium (NBT). ${ }^{30}$ The absorbance change in sample was taken at $560 \mathrm{~nm}$. Catalase (CAT) activity was assessed by estimation of hydrogen peroxide $\left(\mathrm{H}_{2} \mathrm{O}_{2}\right)$ decomposition. ${ }^{31}$ The absorbance change per min was measured at $240 \mathrm{~nm}$. Indirectly malondialdehyde (MDA) content was estimated by using thiobarbituric acid (TBA) and trichloroacetic acid (TCA) reagents. ${ }^{32}$ The absorbance of reaction mixture was taken at $540 \mathrm{~nm}$. The reduced glutathione (GSH) content in lung homogenate was determined. ${ }^{33}$ The absorbance of reaction mixture was taken at $415 \mathrm{~nm}$. The level of nitric oxide (NO) was measured by treated with Griess reagent. ${ }^{34}$ Absorbance of reaction mixture was taken at $550 \mathrm{~nm}$.

Myeloperoxidase (MPO) activity was evaluated by method of Goldblum et al. with slight modification. ${ }^{35}$ Briefly, sample was prepared by homogenizing the lung tissue in $1: 5(\mathrm{w} / \mathrm{v})$ ratio using PBS $(50 \mathrm{mM}, \mathrm{pH} 6)$ containing $0.5 \%(\mathrm{w} / \mathrm{v})$ cetyl trimethylammonium bromide $(\mathrm{CTAB})$ as cationic detergent. Subsequently, mixture was set for repeated freeze-thawed and sonication activity and centrifuged at 15,000 $\mathrm{g}(10 \mathrm{~min})$. For the assessment of MPO activity, $0.1 \mathrm{ml}$ of supernatant was added with $2.9 \mathrm{ml}$ of PBS (50 mM, pH 6) having $0.167 \mathrm{mg} / \mathrm{ml}$ o-dianisidine dihydrochloride and $0.0005 \%(\mathrm{v} / \mathrm{v}) \mathrm{H}_{2} \mathrm{O}_{2}$ with the final reaction mixture volume of $3 \mathrm{ml}$. The change in absorbance per min was measured at $450 \mathrm{~nm}$. Hydroxyproline (HYP) content in lung tissue was estimated by method of Edwards and O'Brien et al. ${ }^{36}$ Briefly, lung tissue dried at $120^{\circ} \mathrm{C}$ for $48 \mathrm{~h} .2 \mathrm{ml}$ of $6 \mathrm{M}$ $\mathrm{HCl}$ was added to the dried lung tissue $(20 \mathrm{mg})$ and kept at $120^{\circ} \mathrm{C}$ for $24 \mathrm{~h}$ for complete acid hydrolysis. $2 \mathrm{ml}$ of PBS was added to reconstitute the sample and 
incubated at $60^{\circ} \mathrm{C}$ for $1 \mathrm{~h}$ by using hot water bath. Subsequently, samples were cooled and centrifuged and supernatant was used to determine HYP content. For HYP assay, $2 \mathrm{ml}$ supernatant was added with $1.0 \mathrm{ml}$ of $50 \mathrm{mM}$ chloramine-T reagent and allowed to precede the reaction for $20 \mathrm{~min}$ at RT. Freshly prepared $1.0 \mathrm{ml}$ Ehrlich's reagent was added and placed the sample in hot water bath at $60^{\circ} \mathrm{C}$ for $15 \mathrm{~min}$. Then, sample was cooled and absorbance was measured at $550 \mathrm{~nm}$. Concentration of test sample was calculated against 4-hydroxy-L-proline standard curve equation.

\section{Oxygen saturation test}

The decreased lung functionality and respiratory insufficiency leads to reduced blood oxygen content and eventually reflects the severity of lung condition. Based on these facts, the assessment of oxygen saturation content in all study animals were carried out by using a non-invasive monitor (Pulse-Oximeter Model: FPO-91, Niscomed Co., Ltd. India) as discussed elsewhere ${ }^{37}$ During measurement of oxygen saturation content, rats were placed in the restrained cage and the sensor clip was applied at the proximal end on ventral tail artery after thoroughly cleaned. Oxygen saturation content was recorded at baseline and thereafter on weekly basis and lastly $2 \mathrm{~h}$ before sacrificed the rats at end of the study. The values of blood oxygen saturation content were expressed in percent (\%).

\section{Survival rate analysis}

A total of 15 rats in each arm (CSS, CNSS, AFC and BLM) were taken in the study of survival analysis. ${ }^{38,39}$ However, in CSS and BLM arms survival rate in the rats were observed for the duration of 21 days, but in CNSS and AFC arms survival rate were observed for the duration of 42 days as per the study duration. The statistical comparison of survival rate among different arms was performed by using log-rank (Mantel-Cox) test. Moreover, the survival rate was expressed as survival percent (\%).

\section{Histopathological examination}

The lungs were quickly removed after sacrificing the study animals and the left lung was kept in 10\% neutral buffered formalin solution for further processing. Formalin-fixed left lung tissues were embedded in paraffin and serial sections were transversely cut at $5 \mu \mathrm{m}$ thickness through microtome. Sections were stained before being examined under light microscope and captured photomicrographs (Canon PowerShot S70, Japan). Hematoxyline and eosin ( $\mathrm{H}$ and $\mathrm{E})$ and Masson's trichrome (MT) staining were performed to observe morphological changes in lung parenchyma and collagen deposition into interstitial spaces respectively. All photomicrographs were assessed through application of Image J software (http://wsr.imagej.net/distros/ win/ij153-win-java8.zip). Degree of inflammation was assessed in $\mathrm{H} \& \mathrm{E}$ stained sections by grading and scoring of inflammatory condition as microscopically observed. The inflammatory condition of lung parenchyma was differentiated and scored according to Szapiel technique. ${ }^{40}$ The semi-quantitative evaluation of MT stained sections were carried out by using Ashcroft method. ${ }^{41}$

\section{Statistical Analysis}

For all parameters, statistical analysis was performed through Graphpad prism Statistical software (V 5.1) by using one-way ANOVA and compared by post-hoc Tukey-Kramer test. However, for survival analysis a log-rank (Mantel-Cox) test was used. All data are presented as mean \pm standard errors of mean (SEM) and the least criterion was set at $p<0.05$ to be considered statistical significance for all comparisons in the study.

\section{RESULTS}

\section{Effect of AFC and BLM on percent change of body weight}

Treatments with AFC resulted in a significant decrease in the body weight compared to the CNSS and AFC+PD arms till 42 days of study possibly due to severe lung injury induced by enhanced oxidative stress. But in AFC+PD arms the body weight continued with non-significant change in comparison to CNSS arm. However, treatment with BLM showed initially marked decrease in body weight as compared to CSS and BLM+PD arms but after 21 days of drug installation, rats were started regaining their body weight towards initial value (Figure 2).

\section{Effect of AFC and BLM on lung/body weight ratio}

In AFC and BLM treated arms, L/B ratios were observed significantly high as compared to CNSS and CSS arm respectively. Similarly, in AFC-42 arm the L/B ratio result was significantly high as compared to BLM-21 indicates relatively more lung inflammatory condition was induced by AFC exposure than BLM instillation. But, in BLM+PD-21 and AFC+PD-42 arms L/B ratios were found significantly low as compared to BLM-21 and AFC-42 arm respectively. However, in AFC+PD-42 $\operatorname{arm~L/B~ratio~was~remained~significantly~high~as~com-~}$ pared to CNSS arm even after treatments with PD (50 $\mathrm{mg} / \mathrm{kg}$ ) (Figure 3), most likely due to repeated insult of lungs tissue through combined risk factors in the duration of study. 


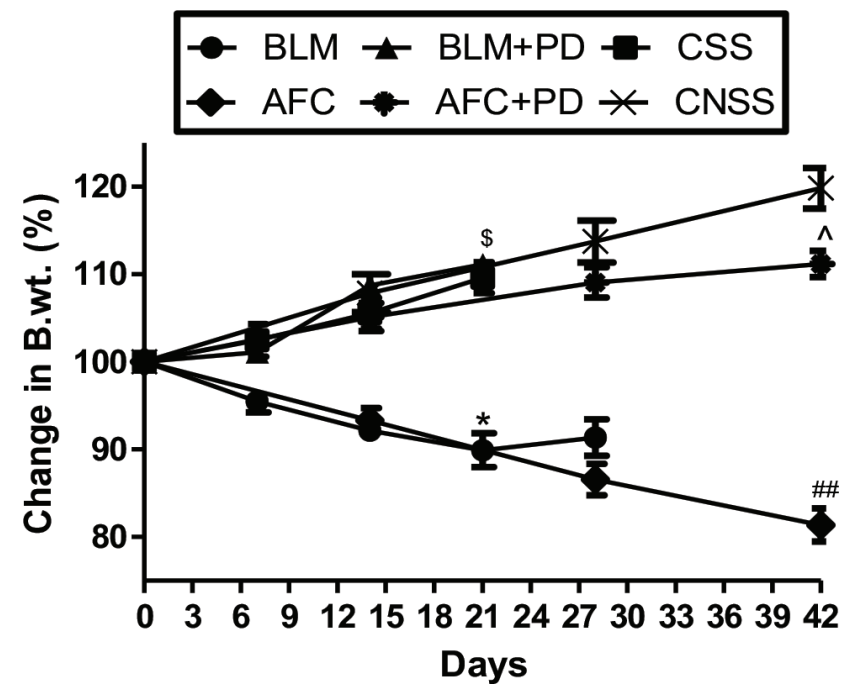

Figure 2: Effect of AFC and BLM treatment with or without PD on percent change of body-weight. " $p<0.05$ BLM vs. CSS, ${ }^{s} p<0.05$ BLM vs. BLM+PD, ${ }^{\prime} p<0.01$ AFC vs. CNSS, ${ }^{\wedge} p<0.05$ $A F C$ vs. AFC+PD. Values are given as Mean $\pm \operatorname{SEM},(n=5)$.

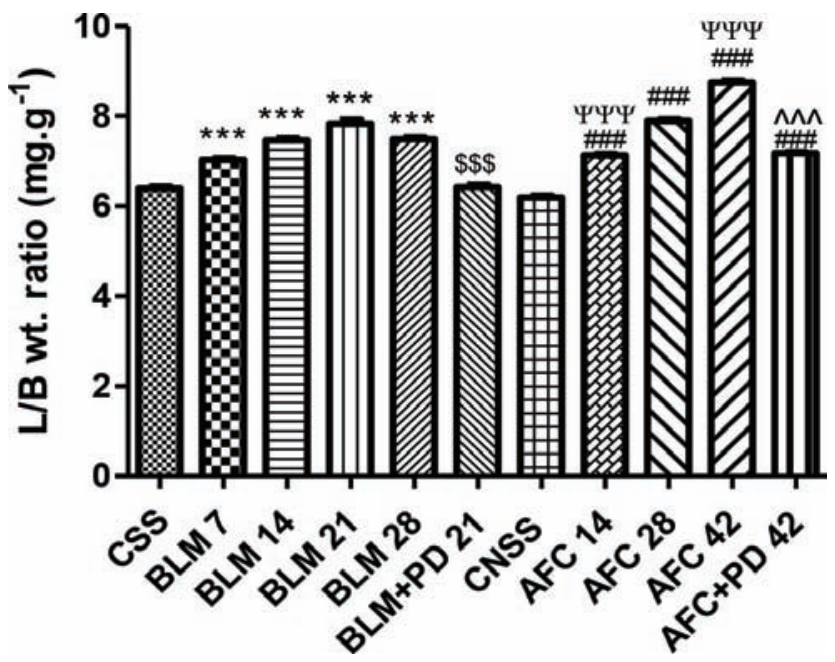

Figure 3: Effect of BLM and AFC treatment with or without PD on lung-body weight ratio. " $p<0.001$ BLM-7, BLM-14, BLM-21 and BLM-28 vs. CSS; $\$ \$ \$ p<001$ BLM-21 vs. BLM+PD-21; $\# \#$ < 0.001 AFC-14, AFC-28, AFC-42 and AFC+PD-42 vs. CNSS; ${ }^{\wedge \wedge} p<0.001$ AFC-42 vs. AFC+PD-42; ${ }^{{ }^{\top} \Psi} p<0.001$ BLM-21 vs. AFC-14 and AFC-42. Values are given as Mean \pm SEM, $(n=5)$.

\section{Effect of AFC and BLM on exercise performance test}

The effect of AFC and BLM treatments on study animals during EPT with respect to distance covered were observed as significantly decreased compared to their respective control CNSS and CSS arm. But, in comparison to BLM-21 and AFC-42 arms, EPT values were significantly increased in BLM+PD-21 and AFC+PD-42 arms respectively (Figure 4), represents somehow restoration of lung condition, which ultimately enhanced performance during the physical exercise.

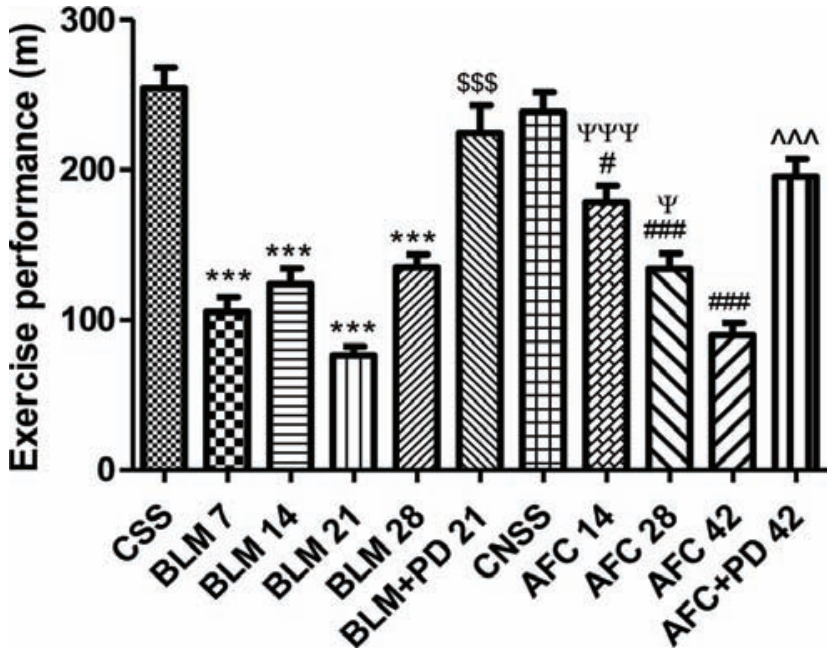

Figure 4: Effect of BLM and AFC treatments with or without PD on physical exercise using forced running wheel. ${ }^{* * *} p<0.001$ BLM-7, BLM-14, BLM-21 and BLM-28 vs. CSS; ${ }^{\$ \$} p<0.001$ BLM-21 vs. BLM+PD-21; ${ }^{*} p<0.05$ AFC-14 vs. CNSS; $p<0.001$ AFC-28 and AFC-42 vs. CNSS; ${ }^{\wedge \wedge \wedge} p<0.001$ AFC-42 vs. AFC+PD-42; ${ }^{4 \psi \psi} p<0.001$ BLM-21 vs. AFC-14 and ${ }^{\Psi} p<0.05$ BLM-21 vs.AFC-28. Values are given as Mean \pm SEM, $(n=5)$.

Effect of AFC and BLM on cells permeation in BALF

The total leukocytes count and its differential values in BALF obtained from different experimental arms are presented in Table 1.

Effect of $A F C$ and BLM on early lung injury markers in BALF

The obtained values of TP, LDH, ALP, CK-MB and TNF- $\alpha$ parameters in BALF samples and their statistical comparison are presented in Table 2.

Effect of AFC and BLM on oxidative-stress, inflammatory and fibrotic markers in lung

Obtained values of GSH, MDA, CAT, SOD, NO parameters and their statistical comparison are presented in Table 3. As compared to CNSS and CSS treated rats, the levels of HYP and MPO were significantly $(p<0.05)$ increase in the lung of all AFC and BLM arms. While, BLM-21 compared to AFC-42 arm, HYP was nonsignificantly different, but, MPO was found significantly high in AFC-42 arm indicates relatively induced advance lung inflammation than BLM-21 arm. PD $(50 \mathrm{mg} / \mathrm{kg})$ treatment exhibited marked inhibition in alterations of HYP and MPO levels induced by insulting with AFC and BLM. However, HYP and MPO levels in AFC+PD-42 arm remained significantly high as compared to CNSS arm even after PD $(50 \mathrm{mg} / \mathrm{kg})$ treatment. It shows insufficiency of PD in complete reversal of 
Table 1: Study of cells permeation in bronchoalveolar lavage fluid (BALF).

\begin{tabular}{|c|c|c|c|c|c|c|}
\hline \multirow{2}{*}{ Study Arms } & \multirow{2}{*}{$\begin{array}{l}\text { Total Cells Count } \\
\times\left(10^{-6} / \mathrm{ml}\right)\end{array}$} & \multicolumn{5}{|c|}{ Differential Leukocytes Count } \\
\hline & & $M(\%)$ & $L(\%)$ & $N(\%)$ & $E(\%)$ & B (\%) \\
\hline css & $0.46 \pm 0.02$ & $83.73 \pm 1.07$ & $11.26 \pm 0.62$ & $2.40 \pm 0.51$ & $2.06 \pm 0.29$ & $0.53 \pm 0.27$ \\
\hline BLM-7 & $1.04 \pm 0.06^{* * t+}$ & $22.53 \pm 1.99^{* * * t}$ & $24.73 \pm 1.86^{* * *+}$ & $48.66 \pm 1.02^{*+*}$ & $3.33 \pm 0.42$ & $0.73 \pm 0.32$ \\
\hline BLM-14 & $0.96 \pm 0.01^{* * *}$ & $30.40 \pm 0.96^{* * *}$ & $20.33 \pm 1.38^{* *}$ & $45.20 \pm 2.05^{* * *}$ & $3.20 \pm 0.58$ & $0.87 \pm 0.39$ \\
\hline BLM-21 & $1.31 \pm 0.04^{* * *}$ & $39.80 \pm 2.32^{* * *}$ & $17.60 \pm 2.27$ & $38.73 \pm 1.91^{* * *}$ & $3.06 \pm 0.68$ & $0.80 \pm 0.37$ \\
\hline BLM-28 & $1.01 \pm 0.06^{* * *+}$ & $53.87 \pm 2.28^{* t+*}$ & $14.80 \pm 1.62$ & $27.60 \pm 1.80^{* * *}$ & $2.93 \pm 0.32$ & $0.80 \pm 0.34$ \\
\hline BLM+PD-21 & $0.53 \pm 0.03^{\$ \$ \$}$ & $75.13 \pm 1.96^{\$ \$ \$}$ & $10.06 \pm 1.33$ & $12.13 \pm 1.24^{* *}, \$ \$$ & $2.00 \pm 0.52$ & $0.66 \pm 0.32$ \\
\hline CNSS & $0.39 \pm 0.01$ & $86.20 \pm 1.35$ & $8.80 \pm 0.92$ & $2.20 \pm 0.37$ & $2.40 \pm 0.54$ & $0.40 \pm 0.19$ \\
\hline AFC-14 & $0.68 \pm 0.03^{\# \#, \psi \psi \psi}$ & $43.93 \pm 2.05^{\# \#}$ & $13.47 \pm 1.38$ & $27.46 \pm 2.32^{\# \#, \psi \psi}$ & $14.27 \pm 2.10^{\# \#, \psi \psi \psi}$ & $0.86 \pm 0.27$ \\
\hline AFC-28 & $1.16 \pm 0.06^{\# \#}$ & $35.73 \pm 3.27^{\# \# \#}$ & $18.80 \pm 2.08^{\# \#}$ & $38.06 \pm 1.58^{\# \# \#}$ & $6.40 \pm 0.58^{\#}$ & $1.00 \pm 0.49$ \\
\hline AFC-42 & $1.53 \pm 0.03^{\# \#, 4}$ & $25.00 \pm 1.39^{\# \#, \psi \psi \psi}$ & $21.87 \pm 1.51^{\# \#}$ & $46.86 \pm 2.74^{\# \# \#}$ & $5.07 \pm 0.32$ & $1.20 \pm 0.37$ \\
\hline AFC+PD-42 & $0.59 \pm 0.03^{\#, \wedge \wedge}$ & $60.06 \pm 3.00^{\# \# \#, \wedge \wedge n}$ & $14.27 \pm 1.81$ & $21.46 \pm 1.74^{\# \#,{ }^{\wedge \wedge \wedge}}$ & $3.33 \pm 0.33$ & $0.86 \pm 0.17$ \\
\hline
\end{tabular}

$\mathrm{M}=$ Macrophage $; \mathrm{L}=$ Lymphocyte; $\mathrm{N}=$ Neutrophil $\mathrm{E}=$ Eosinophil; $\mathrm{B}=$ Basophil. Values are given as Mean $\pm \mathrm{SEM},(n=5)$.

Symbols denoted: BLM vs. CSS (*); BLM-21 vs. BLM+PD-21 (\$); AFC vs. CNSS (\#); AFC-42 vs. AFC+PD-42 (^) and BLM-21 vs. AFC ( $)$ ).

Statistical significance interpreted as: $P<0.05=(*, \$, \#, \wedge, \Psi) ; P<0.01=(* *, \$ \$, \# \#, \wedge \wedge, \Psi \Psi)$ and $P<0.001=(* * *, \$ \$ \$, \# \# \#, \wedge \wedge \wedge, \Psi \Psi \Psi)$.

\begin{tabular}{|c|c|c|c|c|c|}
\hline \multirow{2}{*}{ Study arm } & TP & LDH & ALP & CK-MB & TNF- $\alpha$ \\
\hline & $\left(\mu \mathrm{g} \cdot \mathrm{ml}^{-1}\right)$ & $\left(U . L^{-1}\right)$ & $\left(U . L^{-1}\right)$ & $\left(U . L^{-1}\right)$ & (pg.ml-1) \\
\hline css & $129.67 \pm 3.33$ & $40.00 \pm 0.41$ & $145.14 \pm 4.63$ & $113.74 \pm 1.30$ & $44.15 \pm 1.02$ \\
\hline BLM-7 & $498.61 \pm 9.56^{* * *}$ & $44.03 \pm 1.02$ & $182.59 \pm 8.45^{*}$ & $138.40 \pm 1.26^{* *}$ & $59.02 \pm 1.29^{* *}$ \\
\hline BLM-14 & $867.33 \pm 38.13^{* * *}$ & $48.92 \pm 1.22^{* * *}$ & $224.73 \pm 7.89^{* * *}$ & $163.82 \pm 2.39^{* * *}$ & $75.70 \pm 2.06^{* * *}$ \\
\hline BLM-21 & $1252.83 \pm 28.64^{* * *}$ & $54.07 \pm 1.07^{* * *}$ & $280.91 \pm 5.32^{* * *}$ & $195.98 \pm 3.80^{* * *}$ & $92.30 \pm 1.30^{* * *}$ \\
\hline BLM-28 & $1107.50 \pm 13.29^{* * *}$ & $50.70 \pm 0.87^{* * *}$ & $257.22 \pm 7.16^{* * *}$ & $174.48 \pm 7.94^{* * *}$ & $80.23 \pm 1.03^{* * *}$ \\
\hline BLM+PD-21 & $158.17 \pm 4.80^{\$ \$ \$}$ & $40.70 \pm 1.02^{\$ \$ \$}$ & $157.25 \pm 2.24^{\$ \$ \$}$ & $120.40 \pm 2.85^{\$ \$ \$}$ & $50.79 \pm 1.28^{\$ \$ \$}$ \\
\hline CNSS & $127.65 \pm 4.62$ & $38.96 \pm 1.07$ & $147.89 \pm 7.58$ & $109.74 \pm 0.99$ & $41.81 \pm 1.05$ \\
\hline AFC-14 & $541.67 \pm 2.87^{\# \#, \psi \psi \psi}$ & $46.44 \pm 1.44^{\#, \Psi \psi}$ & $189.75 \pm 4.03^{\#, \Psi \psi \psi}$ & $143.65 \pm 0.66^{\# \#, \Psi \psi \psi}$ & $64.72 \pm 4.93^{\# \#, \psi \psi \psi}$ \\
\hline AFC-28 & $915.34 \pm 6.83^{\# \#, \psi \psi \psi \psi}$ & $51.40 \pm 1.31 \ldots \#$ & $240.42 \pm 11.23^{\# \#, \Psi}$ & $173.48 \pm 2.96^{\# \#, \Psi \psi}$ & $78.19 \pm 1.85^{\# \#, \psi \psi}$ \\
\hline AFC-42 & $1337.68 \pm 12.71 \ldots$ & $60.73 \pm 1.22^{\# \#, \psi}$ & $291.10 \pm 6.72^{\# \#}$ & $226.39 \pm 1.71^{\# \#, \psi \psi \psi}$ & $104.42 \pm 2.43^{\# \#, \psi}$ \\
\hline AFC+PD-42 & $425.84 \pm 13.24^{\# \# \#, \wedge \wedge n}$ & $41.59 \pm 0.33^{\wedge \wedge}$ & $185.2 \pm 1.21^{\#, \wedge \wedge}$ & $132.49 \pm 3.16^{\# \#, \wedge \wedge \wedge}$ & $62.45 \pm 2.98^{\# \#, \wedge \wedge}$ \\
\hline
\end{tabular}

$\mathrm{TP}=$ Total protein; $\mathrm{LDH}=$ Lactate dehydrogenase; $\mathrm{ALP}=$ Alkaline phosphatase; $\mathrm{CK}-\mathrm{MB}=$ Creatinine Kinase; TNF- $\alpha=$ Tumor necrosis factor- $\alpha$ Symbols denoted: BLM vs. CSS $(*)$; BLM-21 vs. BLM+PD-21 (\$); AFC vs. CNSS (\#); AFC-42 vs. AFC+PD-42 $(\wedge)$ and BLM-21 vs. AFC ( $)$ ).

Statistical significance interpreted as: $P<0.05=(*, \$, \#, \wedge, \Psi) ; P<0.01=(* *, \$ \$, \# \#, \wedge \wedge, \Psi \Psi)$ and $P<0.001=(* * *, \$ \$ \$, \# \# \#, \wedge \wedge$, $\Psi \Psi \Psi)$. Values are given as Mean \pm SEM, $(n=5)$

MPO and HYP parameters in AFC+PD-42 treated rats as shown in Figure 5 and 6 respectively.

\section{Effect of AFC and BLM on oxygen saturation percent}

There was no significant difference obtained in the oxygen saturation percent in CNSS and CSS arms. However, when AFC and BLM arms compared with CNSS and CSS arms, there was marked decrease in oxygen saturation percent. But in BLM-7 and BLM+PD-21 arms the level of saturated oxygen remained almost same as compared to CSS arm.
Similarly, between AFC+PD-42 and CNSS arms there was no significant difference observed in oxygen saturation percent. PD $(50 \mathrm{mg} / \mathrm{kg})$ administration in AFC+PD-42 and BLM+PD-21arms significantly increased oxygen saturation percent as compared to AFC-42 and BLM-21 arms respectively (Figure 7).

\section{Effect of AFC and BLM on survival rate}

In AFC and BLM arm the survival rates were found significantly $(p<0.001)$ different. Survival proportion in AFC was obtained as $80.0 \%$ as compared to CNSS arm during 42 days of study span. However, survival 


\begin{tabular}{|c|c|c|c|c|c|}
\hline \multirow{2}{*}{ Study arm } & SOD & CAT & MDA & GSH & No \\
\hline & (U.mg prt ${ }^{-1}$ ) & $\begin{array}{c}\text { (U.min }{ }^{-1} \cdot \mathrm{mg}^{-1} \\
\text { tissue }^{-1} \text { ) }\end{array}$ & (nM.mg prt ${ }^{-1}$ ) & (nM.mg prt ${ }^{-1}$ ) & $\left(\mu \mathrm{M} . \mathrm{mg} \mathrm{prt}^{-1}\right)$ \\
\hline css & $1.84 \pm 0.03$ & $18.26 \pm 0.21$ & $0.08 \pm 0.01$ & $281.20 \pm 3.55$ & $0.60 \pm 0.01$ \\
\hline BLM-7 & $1.64 \pm 0.04$ & $15.28 \pm 0.44$ & $0.25 \pm 0.03^{* * *}$ & $243.08 \pm 3.65^{* * *}$ & $0.73 \pm 0.02^{* * *}$ \\
\hline BLM-14 & $1.46 \pm 0.02^{* * *}$ & $12.05 \pm 0.54^{* * *}$ & $0.42 \pm 0.02^{* * *}$ & $205.22 \pm 4.19^{* * *}$ & $0.88 \pm 0.03^{* * *}$ \\
\hline BLM-21 & $1.31 \pm 0.04^{* * * *}$ & $8.40 \pm 0.40^{* * * *}$ & $0.58 \pm 0.02^{* * *}$ & $163.71 \pm 5.63^{* * *}$ & $1.04 \pm 0.02^{* * * *}$ \\
\hline BLM-28 & $1.38 \pm 0.05^{\text {tw* }}$ & $11.57 \pm 0.29^{* * *}$ & $0.47 \pm 0.01^{\text {t*t* }}$ & $212.79 \pm 2.66^{* * *}$ & $0.95 \pm 0.01^{* * *}$ \\
\hline BLM+PD-21 & $1.77 \pm 0.01^{\$ \$ \$}$ & $17.65 \pm 0.76^{\$ \$ \$}$ & $0.09 \pm 0.01^{\$ \$ \$}$ & $272.59 \pm 6.48^{\$ \$ \$}$ & $0.61 \pm 0.01^{\$ \$ \$}$ \\
\hline CNSS & $1.86 \pm 0.05$ & $20.02 \pm 0.80$ & $0.08 \pm 0.01$ & $285.64 \pm 4.57$ & $0.59 \pm 0.01$ \\
\hline AFC-14 & $1.61 \pm 0.04^{\#, \Psi \psi}$ & $15.95 \pm 0.77^{\#, \psi \psi \psi \psi}$ & $0.25 \pm 0.01 \ldots \#+, \psi \psi \psi$ & $250.65 \pm 4.10^{\#, \psi \psi \psi \psi}$ & $0.75 \pm 0.02^{\# \#, \psi \psi \psi}$ \\
\hline AFC-28 & $1.39 \pm 0.02^{\# \#}$ & $12.54 \pm 0.39^{\# \#, \psi \psi}$ & $0.43 \pm 0.02^{\# \#, \Psi \psi \psi}$ & $209.40 \pm 2.03^{\# \#, \psi \psi \psi \psi}$ & $0.92 \pm 0.01 \ldots \#, \psi \psi \psi$ \\
\hline AFC-42 & $1.13 \pm 0.01^{\# \# \#}$ & $7.91 \pm 0.26^{\# \#}$ & $0.66 \pm 0.02^{\# \#}$ & $142.04 \pm 2.89 \ldots$ & $1.10 \pm 0.02^{\# \#}$ \\
\hline$A F C+P D-42$ & $1.65 \pm 0.04^{\wedge \wedge \wedge}$ & $16.25 \pm 084^{\# \text {,^^ }}$ & $0.23 \pm 0.01^{\# \# \#, \wedge \wedge n}$ & $260.57 \pm 7.31^{\wedge \wedge \wedge}$ & $0.68 \pm 0.01^{\# \wedge \wedge \wedge}$ \\
\hline
\end{tabular}

SOD = Superoxide dismutase $;$ CAT = Catalase; MDA = Malondialdehyde; GSH = Reduced glutathione; $\mathrm{NO}=$ Nitric oxide

Symbols denoted: BLM vs. CSS (*); BLM-21 vs. BLM+PD-21 (\$); AFC vs. CNSS (\#); AFC-42 vs. AFC+PD-42 (^) and BLM-21 vs. AFC ( $)$ ).

Statistical significance interpreted as: $P<0.05=(*, \$, \#, \wedge, \Psi) ; P<0.01=(* *, \$ \$, \# \#, \wedge \wedge, \Psi \Psi)$ and $P<0.001=(* * *, \$ \$ \$, \# \# \#, \wedge \wedge$, $\Psi \Psi \Psi)$. Values are given as Mean \pm SEM, $(n=5)$.

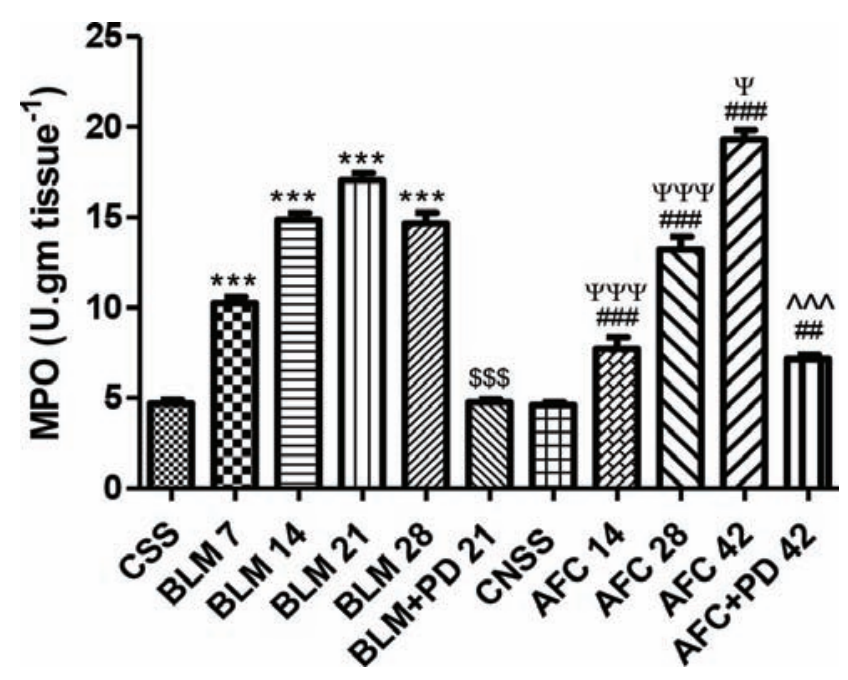

Figure 5: Effect of AFC and BLM treatment with or without PD on MPO activity in lung tissue. ${ }^{* * *} p<0.001$ BLM-7, BLM-14, BLM-21 and BLM-28 vs. CSS; $\$ \$ p<0.001$ BLM-21 vs. BLM+PD-21; $p<0.001$ AFC-14, AFC-28 and AFC-42 vs. CNSS; $\#<0.01$ AFC +PD-42 vs. CNSS; ${ }^{\wedge \wedge} p<0.001$ AFC -42 vs. AFC +PD-42; ${ }^{\Psi \Psi \Psi} p<0.001$ BLM-21 vs. AFC-14 and AFC-28; ${ }^{\Psi} p<0.05$ BLM-21 vs. AFC -42 . Values are given as Mean \pm SEM, $(n=5)$.

proportion in BLM was obtained as $66.67 \%$ as compared to CSS arm in a total span of 21 days. The survival proportion in BLM arm up to 6 days was found as similar as in AFC arm till 42 days (Figure 8). Overall, the survival rate in AFC was markedly increased as

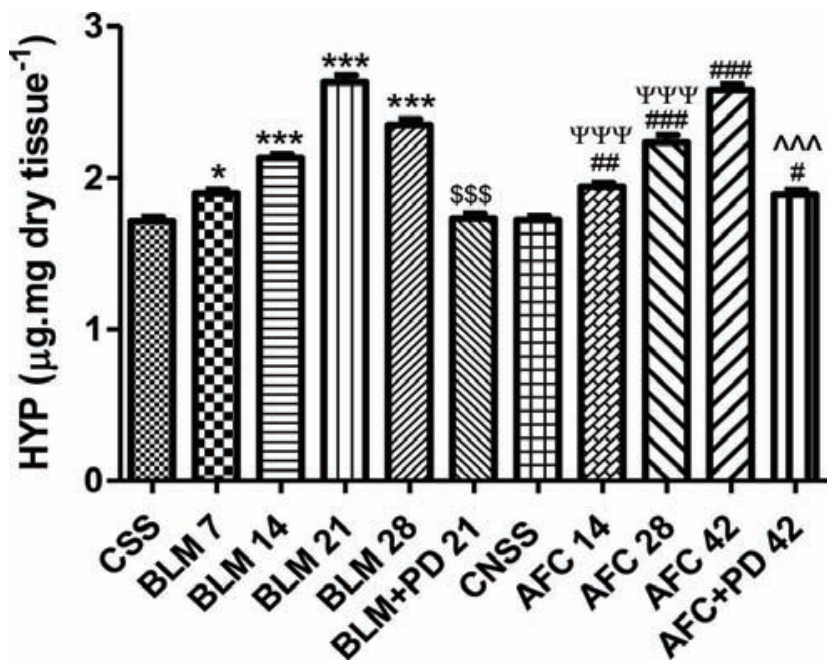

Figure 6: Effect of AFC and BLM treatment with or without PD on HYP content in lung tissue. ${ }^{* * *} p<0.05$ BLM-7 vs. CSS; ${ }^{* * * *} p<0.001$ BLM-14, BLM-21 and BLM-42 vs. CSS; $\$ \$ p<0.001$ $B L M-21$ vs. BLM+PD-21; ${ }^{\#} p<0.01$ AFC-14 vs. CNSS, $\ldots<0.001$ AFC-28 and AFC-42 vs. CNSS; ${ }^{*} p<0.05$ AFC +PD-42 vs. CNSS; ${ }^{\wedge \wedge} p<0.001$ AFC -42 vs. AFC+PD-42; ${ }^{\Psi \Psi \Psi} \boldsymbol{p}<0.001$ BLM-21 vs. AFC-14 and AFC-28. Values are given as Mean \pm SEM, $(n=5)$.

compared to BLM arm. Since, no causality in AFC arm was occurred till first 25 days after initiation of study.

\section{Effect of AFC and BLM on alveolitis score}

The mean values of alveolar inflammation score in lung parenchyma of all arms and their statistical comparison are presented in Table 4. 


\section{Effect of AFC and BLM on fibrosis score}

Ashcroft fibrosis scoring was conducted among rats of different arms and it revealed that in AFC and BLM arms fibrosis score was significantly high as compared to CNSS and CSS arms respectively. However, PD $(50 \mathrm{mg} / \mathrm{kg})$ treatment caused significant reduction in fibrosis score in AFC+PD-42 and BLM+PD-21 arms. But, PD $(50 \mathrm{mg} / \mathrm{kg})$ treatment itself was not effective enough to completely reduce the fibrosis score in AFC+PD-42 as compared to CNSS arm (Figure 9).

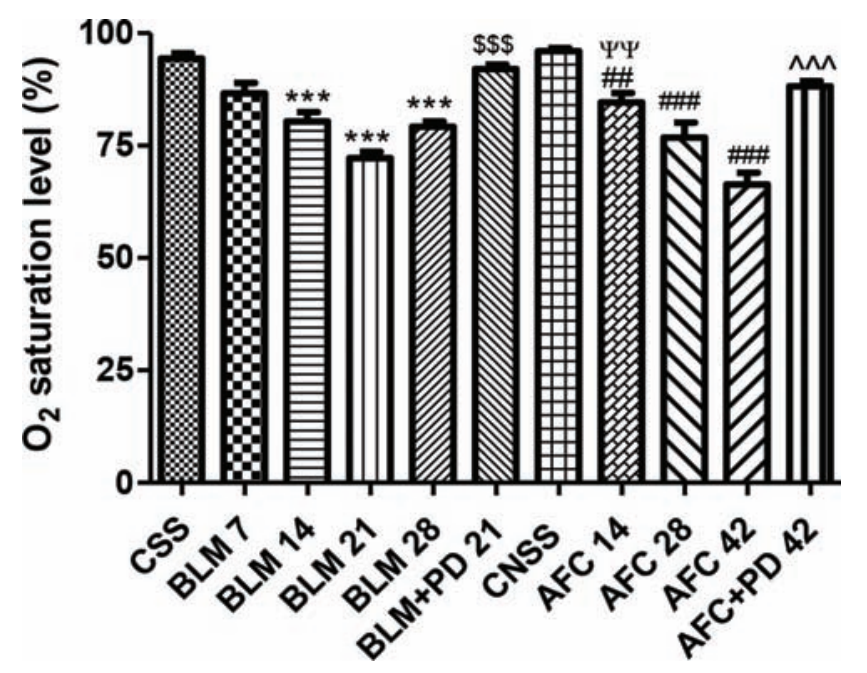

Figure 7: Effect of BLM and AFC treatment with or without PD on peripheral blood saturated oxygen content (\%). ${ }^{* * *} p<0.001$ BLM-14, BLM-21 and BLM-28 vs. CSS; $\$ \$ p<0.001$ BLM-21 vs. $B$ LM+PD-21; $p<0.01$ AFC-14 vs. CNSS; $p<0.001$ AFC-28, $A F C-42$ vs. CNSS; $\wedge^{\wedge} \wedge p<0.001$ AFC -42 vs. AFC +PD-42; ${ }^{\Psi \Psi} p<0.01$ B LM-21 vs. AFC-14. Values are given as Mean \pm SEM, $(n=5)$.

\section{Histopathology of $\mathrm{H}$ and $\mathrm{E}$ and MT stained lung specimen}

$\mathrm{H}$ and $\mathrm{E}$ and MT stained specimen of lungs were studied histologically in all arms. Lungs of rats related to CNSS and CSS arms showed normal architecture of alveoli with regular shape, thin walls, obvious vascular and bronchiolar structures and no evident rupture. But, in the lung sections obtained from AFC-42 and BLM-21 treated rats represented marked infiltration of mono-nucleated cells and alterations in tissue structure accompanied by fibrosis depositions with evident fibrous compression and apparent structural lesion. Including these, collagenous fibers were also observed around the alveoli and bronchioles showing

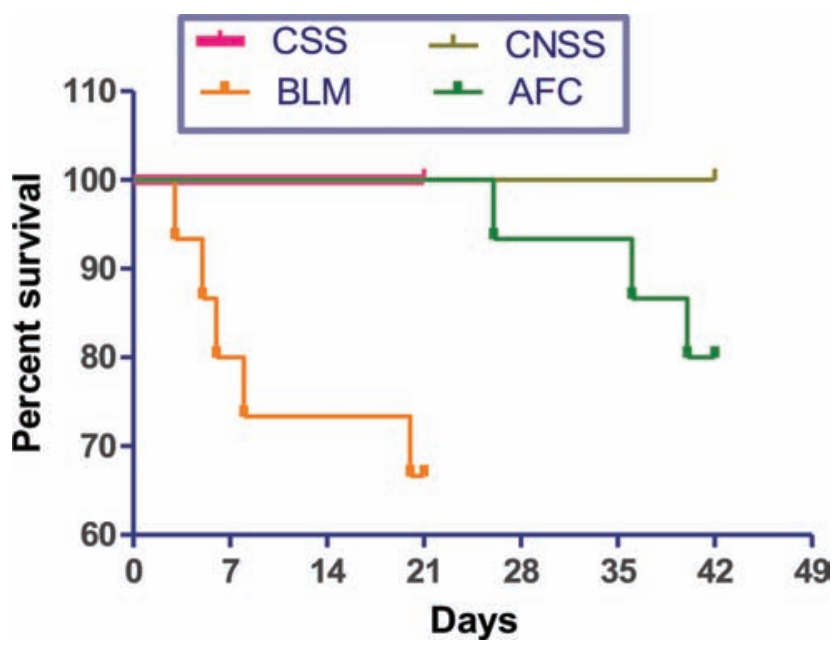

Figure 8: Effect of AFC and BLM treatment on survival percentage. ${ }^{* * *} p<0.001$ B LM vs. CSS; ${ }^{*} p<0.001$ AFC vs. CNSS; ${ }^{\Psi \Psi \Psi} p<0.001$ BLM vs. AFC. $(n=15)$

\begin{tabular}{|c|c|c|c|c|c|c|}
\hline \multirow[b]{2}{*}{ Study Arm } & \multirow{2}{*}{$\begin{array}{l}\text { Number of Animals } \\
\text { (n) }\end{array}$} & \multicolumn{4}{|c|}{ Alveolar Inflammation Score } & \multirow[b]{2}{*}{ Mean \pm SEM } \\
\hline & & $\mathbf{0 x}$ & $1 x$ & $2 \times$ & $3 x$ & \\
\hline css & 5 & 5 & 0 & 0 & 0 & $0.00 \pm 0.00$ \\
\hline BLM-7 & 5 & 0 & 3 & 2 & 0 & $1.40 \pm 0.22^{*}$ \\
\hline BLM-14 & 5 & 0 & 2 & 2 & 1 & $1.80 \pm 0.33^{* *}$ \\
\hline BLM-21 & 5 & 0 & 0 & 2 & 3 & $2.60 \pm 0.22^{* * *}$ \\
\hline BLM-28 & 5 & 0 & 0 & 4 & 1 & $2.20 \pm 0.18^{* * *}$ \\
\hline BLM+PD-21 & 5 & 4 & 1 & 0 & 0 & $0.20 \pm 0.18^{\$ \$ \$}$ \\
\hline CNSS & 5 & 5 & 0 & 0 & 0 & $0.00 \pm 0.00$ \\
\hline AFC-14 & 5 & 0 & 3 & 1 & 1 & $1.60 \pm 0.36$ \\
\hline AFC-28 & 5 & 0 & 1 & 2 & 2 & $2.20 \pm 0.33^{\ldots}$ \\
\hline AFC-42 & 5 & 0 & 0 & 1 & 4 & $2.80 \pm 0.18^{\prime \prime \prime}$ \\
\hline AFC+PD-42 & 5 & 3 & 1 & 1 & 0 & $1.40 \pm 0.22^{\#, \wedge}$ \\
\hline
\end{tabular}

Symbols denoted: BLM vs. CSS (*); BLM-21 vs. BLM+PD-21 (\$); AFC vs. CNSS (\#) and AFC-42 vs. AFC+PD-42 (^).

Statistical significance interpreted as: $P<0.05=(*, \$, \#, \wedge) ; P<0.01=(* *, \$ \$, \# \#, \wedge \wedge)$ and $P<0.001=(* * *, \$ \$ \$, \# \# \#, \wedge \wedge \wedge)$. Values are given as Mean \pm SEM, $(n=5)$. 


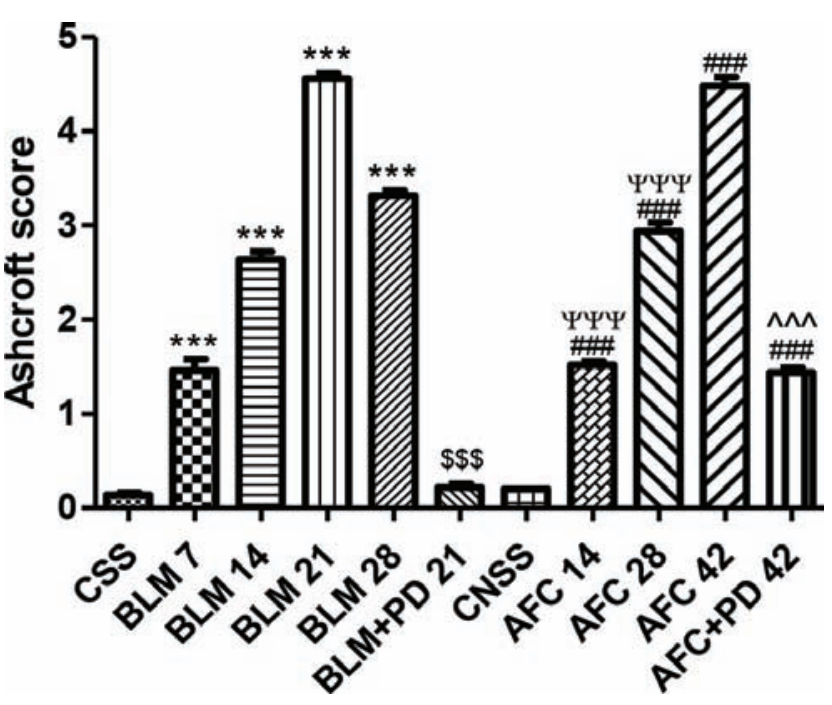

Figure 9: Effect of BLM and AFC treatment with or without PD on Ashcroft fibrotic score. " $" p<0.001$ BLM-7, BLM-14, BLM-21 and BLM-28 vs. CSS; ${ }^{\$ \$} p<0.001$ BLM-21 vs. BLM+PD-21; \#\# $p$ < 001 AFC-14, AFC-28, AFC-42 and AFC+PD-42 vs. CNSS; ${ }^{\wedge \wedge} p<0.001$ AFC-42 vs. AFC+PD-42; ${ }^{\Psi \Psi \Psi} p<0.001$ BLM-21 vs. AFC-14 and AFC-28. Values are given as Mean \pm SEM, $(n=5)$.

materialization of fibrous strands due to enhanced fibrosis. The lungs of rats belong to AFC-14 and BLM-7 arms, exhibited irregularities in shape of alveoli with slight alveolar as well as bronchiolar wall thickening however, no ruptures were seemed in architecture of lungs. Although, the lungs with moderate wall thickening and mild deposition of fibrosis around the alveoli and bronchioles were appeared in the stained lungs section of AFC-28 and BLM-14 treated rats. Treatment with PD $(50 \mathrm{mg} / \mathrm{kg})$ in the AFC+PD-42 and BLM+PD-21 arms, reasonably reduced development of fibrosis also relatively maintained alveolar structure with no alveolar wall thickening as compared to AFC-42 and BLM-21 respectively. But, in AFC+PD-42 treated rats the interstitial space of lungs were still occupied with fibrous structures that indicates incomplete reversal of fibrotic condition as compared with the lungs of CNSS treated rats. Although, the lungs condition of BLM+PD-21 treated rats were remained similar as compared with the lungs of CSS treated rats (Figure 10).

\section{DISCUSSION}

$\mathrm{PF}$ is a prolonged, continuous deteriorating type interstitial lung disease commonly associated with uncontrolled production and deposition of collagen fibers at the venue of persistent lung epithelial cells injury. As a result, it causes transformation of fibroblast into myofibroblast, extra cellular matrix (ECM) remodeling, alveolar structure alteration subsequently alveolar space

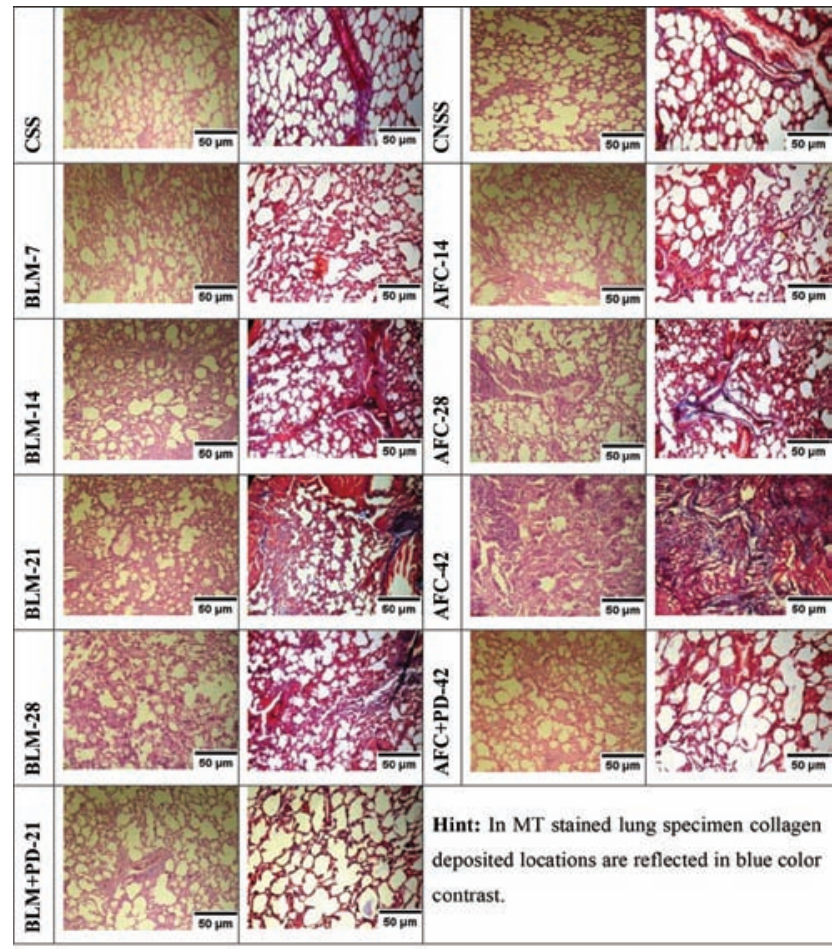

Figure 10: Showing $\mathrm{H}$ and $\mathrm{E}$ and MT stained sections of lungs in different animal arms. CSS and CNSS show normal alveolar structure with no collagen deposition. BLM-7 and AFC-14 show irregular shape of alveoli with slight alveolar and bronchiolar wall thickening. BLM-14 and AFC-28 show moderate wall thickening with mild fibrosis deposition around the alveoli and bronchioles. BLM-21 and AFC-42 show excess collagen deposition with lesion in alveolar wall. However, AFC-42 induced PF reflected uniform and homogenous fibrosis in contrast BLM-21 established patchy type which show auto recovery when compared to BLM-28 lung specimen. BLM+PD-21 and AFC+PD-42 show reversal towards normalization with very less collagen deposition and morphological changes.

reduction that ultimately leading to pulmonary insufficiency and death. Experimental model has a pivotal role in the assessment of disease development. ${ }^{42}$ Currently, single dose intratracheal bleomycin instillation in rodents is broadly acceptable experimental model of PF. As bleomycin induce lung fibrosis spontaneously but, fibrotic condition generally retrograde thereafter. ${ }^{26}$ However, human lung fibrosis usually shows gradual worsening with continuous collagen deposition and develops irreversible condition. Therefore, a new experimental model of PF has been developed through combined risk factors (AFC) shows similarity to human lung fibrosis in various aspects.

In the current investigation, intratracheal administration of bleomycin caused marked reduction in percent body weight post instillation in rats till 3 weeks but after that decreased body weight was started regaining towards normalization. However, pulmonary insult 
through AFC showed significant reduction in percent body weight without reversal tendency. This could be correlated with fibrosis progression phenomenon as decrease in body weight has clinical significance in the evaluation of PF prognosis. ${ }^{43}$ However, pirfenidone treatment assisted considerably to recover the percent loss of body weight. An obvious increase in the lung/body weight ratio, reflected severe lung inflammation as developed with bleomycin and AFC insult and decreases after pirfenidone treatment. But, in AFC+PD-42 arm complete reversal in lung/body weight ratio was not achieved represented development of persistent lung inflammation through AFC exposure. The LDH, CK-MB, ALP and TNF- $\alpha$ are the early lung injury makers and are detectable in BALF after lung tissue damage by means of chemical or mechanical injury. TNF- $\alpha$ is also an important pro-inflammatory cytokine, involved in various cellular interaction and assist in progression of fibrosis. ${ }^{44}$ These lung injury markers were evaluated in the BALF content of rats treated with AFC or bleomycin for different time duration. Bleomycin induced elevated value of $\mathrm{LDH}$, CK-MB, ALP and TNF- $\alpha$ were found with no significant difference between AFC treated rats except that TNF- $\alpha$ level was observed significantly high in AFC-42 as compared to BLM-21 arm, suggested that repeated insult through AFC causes intense elevation of proinflammatory cytokine level which, have close nexus in initiation of PF.

Inflammation is considered a major mechanism in the pathogenesis of PF that particularly involved migration and accumulation leukocytes. These leukocytes in collaboration with AECs could activate localized macrophages. Where, the activated macrophage is considered as the main source to release pro-inflammatory cytokines at injury sites. ${ }^{45,46}$ In this study, repeated insult with AFC and single dose bleomycin instillation set to increased leukocytes permeation in BALF and enhanced oxidative-stress markers including SOD, MDA, CAT, GSH and NO, also inflammatory marker such as MPO and fibrotic marker HYP as hallmark of fibrosis in lung tissue, collectively these leads to deterioration of lung condition, and results in establishment of PF.

Intratracheal, bleomycin instillation facilitates accumulation of collagen fibers in lung parenchymal cells ${ }^{47}$ that usually brought modification in the alveolar wall structures which, compromises with the lungs efficiency during gaseous interchange through alveolar cells membrane. ${ }^{42,48}$ In PF, the decreased lung functionality and respiratory insufficiency leads to reduced oxygen level and eventually reflects the severity of lung fibrosis.
Therefore, physiological evaluation through determination of arterial oxygen saturation is measured as indirect marker of lung condition..$^{49}$ Measurement of percent oxygen saturation by using non-invasive pulse-oximeter reflects vital information about arterial oxygen content. ${ }^{42}$ Earlier experimental studies showed that bleomycin instillation through trachea decreased percent oxygen saturated level in rats. ${ }^{42}$ In this study, similar results were also reflected with intratracheal bleomycin instillation and repeated AFC insult.

The lung mechanics defects and alteration in gaseous exchange are associated with exercise restriction because of dyspnea which is the most noticeable and incapacitating symptom in PF patients. ${ }^{50} \mathrm{PF}$ patient presents marked impairments in physical function and usually measured through 6-min walk test (6-MWT) and considerably decreases during PF severity. ${ }^{26}$ In the present study, severity of PF condition induced by bleomycin instillation and AFC insult in rats were evaluated by means of physical function assessment through EPT. In bleomycin and AFC treated rats, EPT value was observed significantly reduced with respect to control. But, treatment with pirfenidone, exercise performance was effectively revived in both.

The present study has shown that bleomycin instillation and AFC insult drastically affect survival rate in rats. But, as compared to bleomycin model, AFC exhibited enhanced survival ratio primarily due to gradual onset of pulmonary severity. Therefore, AFC induced PF model reflects advantage over bleomycin associated model.

Pulmonary insult by AFC and bleomycin instillation induced histologically marked abnormalities in alveolar structure of lung parenchyma observed under light microscope. These structural changes resemble to the pathological characteristic of clinical PF which shows extreme accumulation of collagen, ECM remodeling compressed alveoli and bronchioles, alveolar wall lesion. ${ }^{19}$ But, pulmonary insult with AFC showed uniform and progressive induction of PF mainly due to thorough and persistent exposure of whole lung during inhalation procedure. In contrast, bleomycin instillation produced patchy type fibrosis that started relapsing mostly after 3 weeks from drug instillation. This finding is in accordance to the previous study. ${ }^{26}$ However, treatment with pirfenidone inhibited both bleomycin as well as AFC induced abnormalities in lung architecture, which was revealed by minimizing the collagen deposition in alveolar wall, peribronchial and interstitial space of lung parenchyma. 


\section{CONCLUSION}

This investigation revealed the significant role of formaldehyde and cigarette smoke in reinforcement of pulmonary fibrosis predominantly during lung inflammatory condition. This study also provides a novel idea to induce homogeneous and progressive pulmonary fibrosis experimental model through combined risk factors which could mimic human lung fibrosis more specifically than induced by bleomycin. Additionally, establishment of progressive lung fibrosis in experimental animals would assist in effective screening of active compounds which may potentially use to treat the human lung fibrosis.

\section{ACKNOWLEDGEMENT}

Author(s) wish to thank and acknowledge Birla Institute of Technology-Mesra, Ranchi Jharkhand, India. For their infrastructural help and support during the conduct of experimental study and providing technical assistance during preparation of this manuscript.

\section{CONFLICT OF INTEREST}

The authors declare that there is no conflict of interest.

\section{ABBREVIATIONS}

AFC: Asthma+Formaldehyde+Cigarette smoke; BLM: Bleomycin; PF: Pulmonary fibrosis; OVA: Ovalbumin; BALF: Bronchoalveolar lavage fluid; MPO: Myeloperoxidase; HYP: Hydroxyproline; MDA: Malondialdehyde; NO: Nitric oxide; TNF- $\alpha$ : Tumor necrosis factor- $\alpha$; CK-MB: Creatine Kinase-MB; ALP: Alkaline phosphatase; LDH: Lactate Dehydrogenase; TLC: Total leukocyte count; DLC: Differential leukocyte count; TP: Total protein; SOD: Superoxide Dismutase; GSH: Reduced Glutathione; CAT: Catalase; CSS: Control with survival surgery; CNSS: Control with no survival surgery; PD: Pirfenidone; CS: Cigarette smoke; FS: Formaldehyde solution; NS: Normal saline; EPT: Exercise Performance Test; ANOVA: Analysis of variance; ECM: Extra cellular matrix.

\section{REFERENCES}

1. Wynn TA. Cellular and molecular mechanisms of fibrosis. J Pathol. 2008;214(2):199-210. doi: 10.1002/path.2277, PMID 18161745.

2. Johannson KA, Balmes JR, Collard HR. Air pollution exposure: a novel environmental risk factor for interstitial lung disease? Chest. 2015;147(4):1161-7. doi: 10.1378/chest.14-1299, PMID 25846532.

3. Kalchiem-Dekel O, Galvin JR, Burke AP, Atamas SP, Todd NW. Interstitial lung disease and pulmonary fibrosis: a practical approach for general medicine physicians with focus on the medical history. J Clin Med. 2018;7(12):476. doi: 10.3390/jcm7120476, PMID 30477216.
4. Fernández Fabrellas E, Peris Sánchez R, Sabater Abad C, Juan Samper G. Prognosis and follow-up of idiopathic pulmonary fibrosis. Med Sci (Basel). 2018;6(2):51. doi: 10.3390/medsci6020051, PMID 29904028.

5. Oh CK, Murray LA, Molfino NA. Smoking and idiopathic pulmonary fibrosis. Pulm Med. 2012;2012:808260. doi: 10.1155/2012/808260.

6. Awadalla NJ, Hegazy A, Elmetwally RA, Wahby I. Occupational and environmental risk factors for idiopathic pulmonary fibrosis in Egypt: a multicenter case-control study. Int J Occup Environ Med (The IJOEM). 2012;3(3):107-16. PMID 23022860.

7. Maher TM, Strek ME. Antifibrotic therapy for idiopathic pulmonary fibrosis: time to treat. Respir Res. 2019;20(1):205. doi: 10.1186/s12931-019-1161-4, PMID 31492155.

8. Degryse AL, Tanjore $\mathrm{H}, \mathrm{Xu} X \mathrm{C}$, Polosukhin VV, Jones BR, McMahon FB, Gleaves LA, Blackwell TS, Lawson WE Repetitive intratracheal bleomycin models several features of idiopathic pulmonary fibrosis. Am J Physiol Lung Cell Mol Physiol. 2010;299(4):L442-52. doi: 10.1152/ajplung.00026.2010. PMID 20562227.

9. Moeller A, Ask K, Warburton D, Gauldie J, Kolb M. The bleomycin animal model: a useful tool to investigate treatment options for idiopathic pulmonary fibrosis? Int J Biochem Cell Biol. 2008;40(3):362-82. doi: 10.1016/j. biocel.2007.08.011, PMID 17936056.

10. Conti S, Harari S, Caminati A, Zanobetti A, Schwartz JD, Bertazzi PA, Cesana G, Madotto $F$. The association between air pollution and the incidence of idiopathic pulmonary fibrosis in Northern Italy. Eur Respir J. 2018;51(1). doi: 10.1183/13993003.00397-2017, PMID 29371377.

11. Manisalidis I, Stavropoulou E, Stavropoulos A, Bezirtzoglou E. Environmental and health impacts of air pollution: a review. Front Public Health. 2020;8:14. doi: 10.3389/fpubh.2020.00014, PMID 32154200.

12. Franz AW, Kronemayer H, Pfeiffer D, Pilz RD, Reuss G, Disteldorf W, et al. Formaldehyde. Ullmann's Encycl Ind Chem. 2016:1-34.

13. Murta GL, Campos KKD, Bandeira ACB, Diniz MF, Costa Gde P, Costa DC, Talvani A, Lima WG, Bezerra FS. Oxidative effects on lung inflammatory response in rats exposed to different concentrations of formaldehyde. Environ Pollut. 2016;211:206-13. doi: 10.1016/j.envpol.2015.12.054, PMID 26774767.

14. Leal MP, Brochetti RA, Ignácio A, Câmara NOS, da Palma RK, de Oliveira LVF, de Fátima Teixeira da Silva D, Lino-Dos-Santos-Franco A. Effects of formaldehyde exposure on the development of pulmonary fibrosis induced by bleomycin in mice. Toxicol Rep. 2018;5:512-20. doi: 10.1016/j. toxrep.2018.03.016, PMID 29854623.

15. Lino-dos-Santos-Franco $A$, Correa-Costa $M$, Durão $A C$, de Oliveira $A P$, Breithaupt-Faloppa AC, Bertoni Jde A, Oliveira-Filho RM, Câmara NO, Marcourakis T, Tavares-de-Lima WFormaldehyde induces lung inflammation by an oxidant and antioxidant enzymes mediated mechanism in the lung tissue. Toxicol Lett. 2011;207(3):278-85. doi: 10.1016/j.toxlet.2011.09.026, PMID 21983654.

16. Zhou LL, Wang M, Liu F, Lu YZ, Song LJ, Xiong L, Xiang F, He XL, Shuai SY, Xin JB, Ye H, Yu F, Ma WL. Cigarette smoking aggravates bleomycin-induced experimental pulmonary fibrosis. Toxicol Lett. 2019;303:1-8. doi: 10.1016/j. toxlet.2018.12.008, PMID 30572104.

17. Casset A, Marchand C, Purohit A, Le Calve S, Uring-Lambert B, Donnay C, Meyer $\mathrm{P}$, de Blay F. Inhaled formaldehyde exposure: effect on bronchial response to mite allergen in sensitized asthma patients. Allergy. 2006;61(11):1344-50. doi: 10.1111/j.1398-9995.2006.01174.x, PMID 17002712.

18. Mittal M, Siddiqui MR, Tran K, Reddy SP, Malik AB. Reactive oxygen species in inflammation and tissue injury. Antioxid Redox Signal. 2014;20(7):1126-67. doi: 10.1089/ars.2012.5149, PMID 23991888.

19. Sriram N, Kalayarasan S, Sudhandiran G. Epigallocatechin-3-gallate exhibits anti-fibrotic effect by attenuating bleomycin-induced glycoconjugates, lysosomal hydrolases and ultrastructural changes in rat model pulmonary fibrosis. Chem Biol Interact. 2009;180(2):271-80. doi: 10.1016/j. cbi.2009.02.017, PMID 19497426.

20. Song X, Yu W, Guo F. Pirfenidone suppresses bleomycin-induced pulmonary fibrosis and periostin expression in rats. Exp Ther Med. 2018;16(3):1800-6. doi: 10.3892/etm.2018.6378, PMID 30186404.

21. Conrad ML, Yildirim AO, Sonar SS, Kılıç A, Sudowe S, Lunow M, Teich R, Renz H, Garn H. Comparison of adjuvant and adjuvant-free murine 
experimental asthma models. Clin Exp Allergy. 2009;39(8):1246-54. doi: 10.1111/j.1365-2222.2009.03260.x, PMID 19438585.

22. Mizuno $M$, Sakaguchi $K$, Sakane I. Oral administration of fucoidan can exert anti-allergic activity after allergen sensitization by enhancement of galectin-9 secretion in blood. Biomolecules. 2020;10(2):258. doi: 10.3390/ biom10020258, PMID 32050429.

23. Abril-Gil M, Garcia-Just A, Pérez-Cano FJ, Franch À, Castell M. Development and characterization of an effective food allergy model in Brown Norway rats. PLOS ONE. 2015;10(4):e0125314. doi: 10.1371/journal.pone.0125314, PMID 25923134.

24. Bradford MM. A rapid and sensitive method for the quantitation of microgram quantities of protein utilizing the principle of protein-dye binding. Anal Biochem. 1976;72(1-2):248-54. doi: 10.1006/abio.1976.9999, PMID 942051.

25. Chen L, Zhao W. Apigenin protects against bleomycin-induced lung fibrosis in rats. Exp Ther Med. 2016;11(1):230-4. doi: 10.3892/etm.2015.2885, PMID 26889245.

26. Schafer MJ, White TA, lijima K, Haak AJ, Ligresti G, Atkinson EJ, Oberg AL, Birch J, Salmonowicz H, Zhu Y, Mazula DL, Brooks RW, FuhrmannStroissnigg H, Pirtskhalava T, Prakash YS, Tchkonia T, Robbins PD, Aubry MC, Passos JF, Kirkland JL, Tschumperlin DJ, Kita H, LeBrasseur NK. Cellular senescence mediates fibrotic pulmonary disease. Nat Commun. 2017;8:14532. doi: 10.1038/ncomms14532, PMID 28230051.

27. Geng X, Dufu K, Hutchaleelaha A, Xu Q, Li Z, Li CM, Patel MP, Vlahakis N, Lehrer-Graiwer J, Oksenberg D. Increased hemoglobin-oxygen affinity ameliorates bleomycin-induced hypoxemia and pulmonary fibrosis. Physiol Rep. 2016;4(17):e12965. doi: 10.14814/phy2.12965, PMID 27624688.

28. Verma R, Kushwah L, Gohel D, Patel M, Marvania T, Balakrishnan S. Evaluating the ameliorative potential of quercetin against the bleomycininduced pulmonary fibrosis in Wistar rats. Pulm Med. 2013;2013:921724. doi: 10.1155/2013/921724

29. Behnia R, Molteni A, Waters CM, Panos RJ, Ward WF, Schnaper HW, TS'Ao CH. Early markers of ventilator-induced lung injury in rats. Ann Clin Lab Sci. 1996;26(5):437-50. PMID 8879362.

30. Kakkar P, Das B, Viswanathan PN. A modified spectrophotometric assay of superoxide dismutase. Indian J Biochem Bio. 1984;21(2):130-2. doi: 10.1006/ abio.1995.0014, PMID 6490072.

31. Cohen G, Dembiec D, Marcus J. Measurement of catalase activity in tissue extracts. Anal Biochem. 1970;34(1):30-8. doi: 10.1016/0003-2697(70)90083-7, PMID 5440916.

32. Ohkawa $\mathrm{H}$, Ohishi N, Yagi K. Assay for lipid peroxides in animal tissues by thiobarbituric acid reaction. Anal Biochem. 1979;95(2):351-8. doi: 10.1016/0003-2697(79)90738-3, PMID 36810.

33. Jollow DJ, Mitchell JR, Zampaglione NA, Gillette JR. Bromobenzeneinduced liver necrosis. Protective role of glutathione and evidence for 3 , 4-bromobenzene oxide as the hepatotoxic metabolite. Pharmacology. 1974;11(3):151-69. doi: 10.1159/000136485, PMID 4831804.

34. Menaka KB, Ramesh A, Thomas B, Kumari NS. Estimation of nitric oxide as an inflammatory marker in periodontitis. J Indian Soc Periodontol. 2009;13(2):75-8. doi: 10.4103/0972-124X.55842, PMID 20407654.

35. Goldblum SE, Wu KM, Jay M. Lung myeloperoxidase as a measure of pulmonary leukostasis in rabbits. J Appl Physiol (1985). 1985;59(6):1978-85. doi: 10.1152/jappl.1985.59.6.1978, PMID 3001018.

36. Edwards CA, O'Brien Jr WD. Modified assay for determination of hydroxyproline in a tissue hydrolyzate. Clin Chim Acta. 1980;104(2):161-7. doi: 10.1016/0009-8981(80)90192-8, PMID 7389130.

37. Zhou Z, Kandhare AD, Kandhare AA, Bodhankar SL. Hesperidin ameliorates bleomycin-induced experimental pulmonary fibrosis via inhibition of TGF-
beta1/Smad3/AMPK and IkappaBalpha/NF-kappaB pathways. Excli J. 2019;18:723-45. doi: 10.17179/excli2019-1094, PMID 31611754.

38. Li H, Hao Y, Zhang H, Ying W, Li D, Ge Y, Ying B, Cheng B, Lian Q, Jin S. Posttreatment with Protectin DX ameliorates bleomycin-induced pulmonary fibrosis and lung dysfunction in mice [sci rep:46754]. Sci Rep. 2017;7:46754. doi: 10.1038/srep46754, PMID 28466866.

39. Tajima S, Bando M, Ishii Y, Hosono T, Yamasawa H, Ohno S, Takada T, Suzuki E, Gejyo F, Sugiyama Y. Effects of edaravone, a free-radical scavenger, on bleomycin-induced lung injury in mice. Eur Respir J. 2008;32(5):1337-43. doi: 10.1183/09031936.00164407, PMID 18614556.

40. Szapiel SV, Elson NA, Fulmer JD, Hunninghake GW, Crystal RG. Bleomycininduced interstitial pulmonary disease in the nude, athymic mouse. Am Rev Respir Dis. 1979;120(4):893-9. doi: 10.1164/arrd.1979.120.4.893, PMID 92208.

41. Ashcroft T, Simpson JM, Timbrell V. Simple method of estimating severity of pulmonary fibrosis on a numerical scale. J Clin Pathol. 1988;41(4):467-70. doi: 10.1136/jcp.41.4.467, PMID 3366935.

42. Kandhare AD, Bodhankar SL, Mohan V, Thakurdesai PA. Effect of glycosides based standardized fenugreek seed extract in bleomycin-induced pulmonary fibrosis in rats: decisive role of Bax, Nrf2, NF-kB, Muc5ac, TNF- $\alpha$ and IL-1 $\beta$. Chem Biol Interact. 2015;237:151-65. doi: 10.1016/j.cbi.2015.06.019, PMID 26093215.

43. Nakatsuka Y, Handa T, Kokosi M, Tanizawa K, Puglisi S, Jacob J, Sokai A, Ikezoe K, Kanatani KT, Kubo T, Tomioka H, Taguchi Y, Nagai S, Chin K, Mishima M, Wells AU, Hirai T. The clinical significance of body weight loss in idiopathic pulmonary fibrosis patients. Respiration. 2018;96(4):338-47. doi: 10.1159/000490355, PMID 30130749.

44. Bale S, Venkatesh P, Sunkoju M, Godugu C. An Adaptogen: Withaferin A Ameliorates in vitro and in vivo Pulmonary Fibrosis by Modulating the Interplay of Fibrotic, Matricelluar Proteins, and Cytokines. Front Pharmacol. 2018;9:248. doi: 10.3389/fphar.2018.00248, PMID 29623041.

45. Lakari E, Pylkäs P, Pietarinen-Runtti P, Pääkkö P, Soini Y, Kinnula VL. Expression and regulation of hemeoxygenase 1 in healthy human lung and interstitial lung disorders. Hum Pathol. 2001;32(11):1257-63. doi: 10.1053/ hupa.2001.28937, PMID 11727267.

46. Yang YL, Hsu HT, Wang KH, Wang CS, Chen CM, Ko WC. Hesperidin-3'o-methylether is more potent than hesperidin in phosphodiesterase inhibition and suppression of ovalbumin-induced airway hyperresponsiveness. Evid Based Complement Alternat Med. 2012;2012:908562. doi: 10.1155/2012/908562.

47. Della Latta V, Cecchettini A, Del Ry S, Morales MA. Bleomycin in the setting of lung fibrosis induction: from biological mechanisms to counteractions. Pharmacol Res. 2015;97:122-30. doi: 10.1016/j.phrs.2015.04.012, PMID 25959210.

48. Liu JF, Nie XC, Shao YC, Su WH, Ma HY, Xu XY. Bleomycin suppresses the proliferation and the mobility of human gastric cancer cells through the smad signaling pathway. Cell Physiol Biochem. 2016;40(6):1401-9. doi: 10.1159/000453192, PMID 27997887.

49. 49. Collins JA, Rudenski A, Gibson J, Howard L, O'Driscoll R. Relating oxygen partial pressure, saturation and content: the haemoglobin-oxygen dissociation curve. Breathe (Sheff). 2015;11(3):194-201. doi: 10.1183/20734735.001415, PMID 26632351.

50. 50. Triantafillidou C, Manali E, Lyberopoulos P, Kolilekas L, Kagouridis K, Gyftopoulos S, Vougas K, Kotanidou A, Alchanatis M, Karakatsani A, Papiris SA. The role of cardiopulmonary exercise test in IPF prognosis. Pulm Med. 2013;2013:514817. doi: 10.1155/2013/514817. 


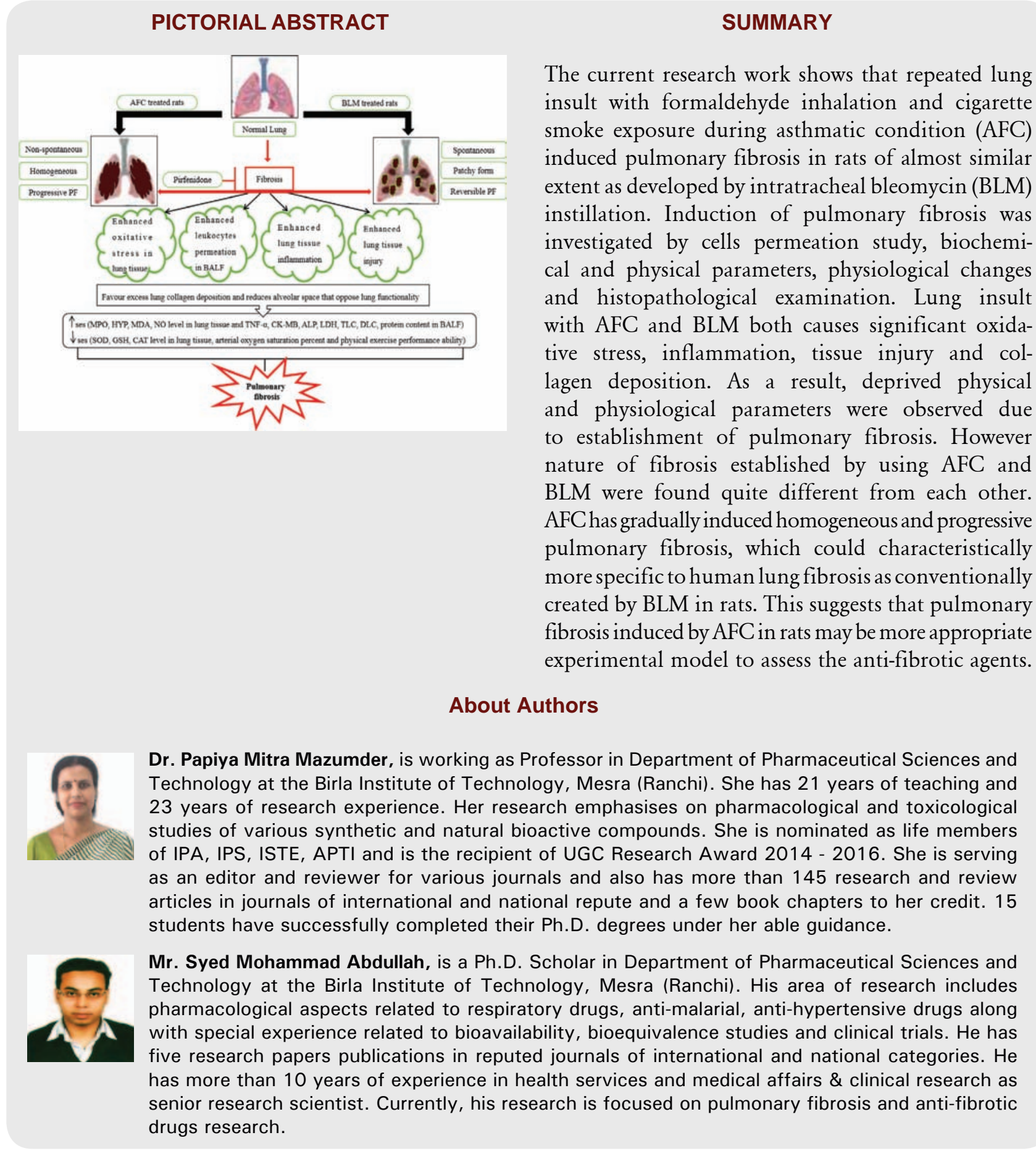

Cite this article: Abdullah SM, Mazumder PM. An Experimental Model to Induce Homogeneous and Progressive Pulmonary Fibrosis in Rats. Indian J of Pharmaceutical Education and Research. 2021;55(3s):s751-s764. 